\title{
The Delay in Confirming COVID-19 Cases Linked to a Religious Group in Korea
}

\author{
Hyung-Ju Kim, Hyun-Seong Hwang, Yong-Hyuk Choi, Hye-Yeon Song, Ji-Seong Park, Chae-Young Yun, Sukhyun Ryu \\ Department of Preventive Medicine, Konyang University College of Medicine, Daejeon, Korea
}

Objectives: As of March 3, 2020, the Shincheonji religious group accounted for the majority of Korean cases of coronavirus disease 2019 (COVID-19). Nonetheless, the most likely cause of the broad spread of COVID-19 among members of the Shincheonji religious group remains largely unknown.

Methods: We obtained data of laboratory-confirmed cases related to the Shincheonji religious group from press releases by Korean public health authorities and news reports. We measured the period from the date of illness onset to the date of COVID-19 confirmation.

Results: We analysed data from 59 cases (median age, 30 years). The estimated median period between the date of symptom onset and the date of COVID-19 confirmation was 4 days (95\% confidence interval, 1-12).

Conclusions: There was a delay in COVID-19 confirmation from the date of illness onset among the cases linked to the Shincheonji religious group. This delay likely contributed to the occurrence of many cases of COVID-19 in the group.

Key words: Coronavirus, Transmission, Religious belief, Korea

\section{INTRODUCTION}

On January 20, 2020, the first case of coronavirus disease 2019 (COVID-19) in Korea was identified in a Chinese person who travelled from Wuhan, China [1]. On February 18, 2020, the first case related to the Shincheonji religious group in Daegu was identified, following which massive contact tracing with an active screening program was implemented for that group. As the number of laboratory-confirmed cases of COVID-19 increased among members of the Shincheonji religious group, the Korean public health authorities announced a serious cri-

Received: March 24, 2020 Accepted: April 8, 2020

Corresponding author: Sukhyun Ryu, MD, PhD

Department of Preventive Medicine, Konyang University College of

Medicine, 158 Gwanjeodong-ro, Seogu, Daejeon 35365, Korea

E-mail: gentryu@onehealth.or.kr

This is an Open Access article distributed under the terms of the Creative Commons Attribution Non-Commercial License (https://creativecommons.org/licenses/bync/4.0/) which permits unrestricted non-commercial use, distribution, and reproduction in any medium, provided the original work is properly cited. sis alert on February 23, 2020. As of March 3, 2020, Korea had the second highest number of confirmed cases of COVID-19 following China.

Given the high proportion of cases related to the Shincheonji religious group among all Korean cases (2992 of 5621 as of March 3, 2020) [2], we conducted a preliminary investigation of the time delay from illness onset to COVID-19 confirmation among members of the group.

\section{METHODS}

\section{Data Sources}

Data were obtained from the daily COVID-19 situation reports from the Korea Centers for Disease Control and Prevention, municipal or provincial departments of public health, and from news reports [2]. The epidemiological information of interest included cases' year of birth, sex, date of illness onset, and origin of infection, including contact history with other laboratory-confirmed cases. We excluded cases with no re- 
ported symptoms and cases not linked to the Shincheonji religious group. We extracted data on laboratory-confirmed cases using a structured data-extraction form.

\section{Statistical Analysis}

We constructed epidemic curves using the date of illness onset and the date of COVID-19 confirmation. Furthermore, we estimated the time delay between the date of illness onset and the date of COVID-19 confirmation by fitting log-normal,

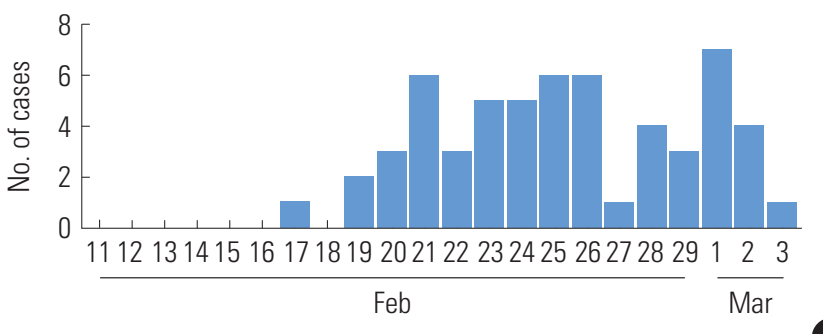

Date of confirmation

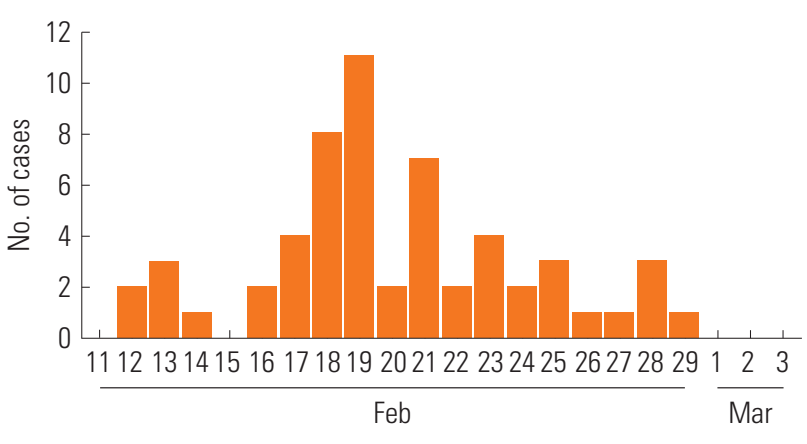

Date of illness onset

B

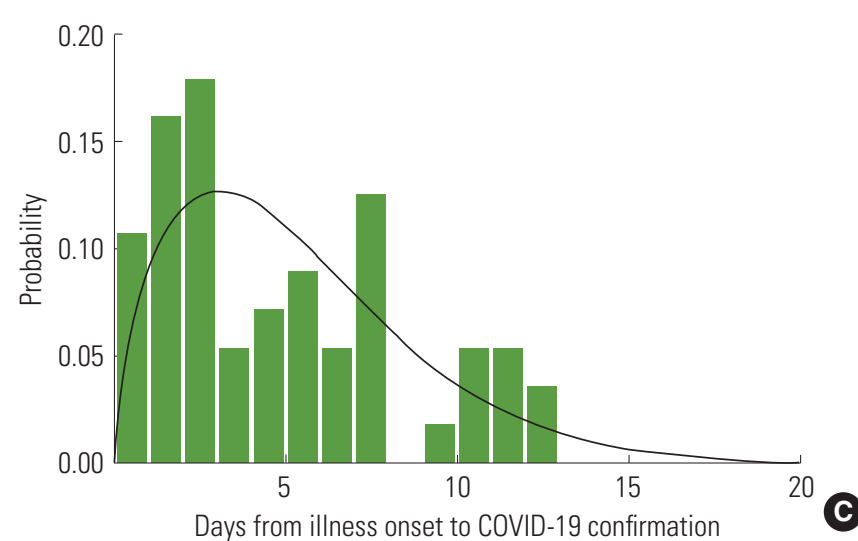

Figure 1. The time delay of coronavirus disease 2019 (COVID-19) infections related to the Shincheonji religious group in Korea, as of March 3, 2020. (A) Curve according to the date of laboratory confirmation ( $n=57)$. (B) Curve according to the date of illness onset for confirmed cases. (C) The estimated time interval for the confirmation of COVID-19 in laboratoryconfirmed cases using the gamma distribution. gamma, and Weibull distributions using the available data [3]. We identified probabilistic distribution models and evaluated the suitability of the distribution models through the Bayesian information criterion $(\mathrm{BIC})$ prior to the statistical analysis $[4,5]$. All statistical analyses were performed using $R$ version 3.0.2 (https://cran.r-project.org/) and the R package "MASS" was used to identify the best model fit.

\section{Ethics Statement}

The need for ethical approval for this study was not necessary, based on the Enforcement Rule of Bioethics and Safety Act No. 13.

\section{RESULTS}

We obtained a publicly available line list of 57 laboratoryconfirmed cases reported between February 20, 2020 and March 3, 2020. The number of cases, as assessed by the date of illness onset, peaked on February 19, 2020 and decreased thereafter (Figure 1A, 1B). Twenty-four cases (42.1\%) were male, and the mean age was 37 years (range, 14-77) (Table 1).

Overall, symptoms were reported in 51 cases. The reported symptoms included cough ( 23 cases; $45.0 \%$ of the sample population), fever (12 cases; $23.5 \%$ ), sore throat (12 cases; $23.5 \%)$, myalgia (11 cases; $21.6 \%)$, headache ( 2 cases; $3.9 \%)$, rhinorrhoea (2 cases; $3.9 \%)$, and chill (2 cases; $3.9 \%)$. The average period between the date of illness onset and the date of COVID-19 confirmation was 5.2 days, with a median of 4 days (range, 0-13).

Table 1. Demographic characteristics of the laboratoryconfirmed cases of coronavirus disease 2019 infections associated with the Shincheonji religious group in Korea, as of March 3, $2020(n=57)$

\begin{tabular}{lc}
\hline Characteristics & $\mathbf{n}(\%)$ \\
\hline Sex & \\
Male & $24(42.1)$ \\
Female & $33(57.9)$ \\
Age (y) & \\
$10-19$ & $4(7.0)$ \\
$20-29$ & $26(45.6)$ \\
$30-39$ & $4(7.0)$ \\
$40-49$ & $9(15.8)$ \\
$50-59$ & $8(14.0)$ \\
$60-69$ & $5(8.8)$ \\
$70-79$ & $1(1.7)$ \\
\hline
\end{tabular}


Table 2. Estimated distribution of the time interval from illness onset to coronavirus disease 2019 confirmation among cases in the Shincheonji religious group in Korea

\begin{tabular}{lccc}
\hline & \multicolumn{3}{c}{ Estimated distribution } \\
\cline { 2 - 4 } & Weibull & Log-normal & Gamma \\
\hline Mean \pm SD $(95 \% \mathrm{CI})$ & $5.3 \pm 1.5$ & $5.5 \pm 4.8$ & $5.3 \pm 3.7$ \\
& $(0.8,12.0)$ & $(1.2,14.4)$ & $(1.0,12.4)$ \\
BIC & 293.0 & 294.6 & 292.7 \\
\hline
\end{tabular}

SD, standard deviation; $\mathrm{Cl}$, confidence interval; BIC, Bayesian information criterion.

The period of time delay was best fit by the gamma distribution (BIC=292.7). Based on our model fit to the distribution, the estimated mean time delay was 5.3 days, with a $95 \%$ confidence interval of 1.0 -12.4 days (Figure 1C, Table 2).

\section{DISCUSSION}

We investigated the cases of COVID-19 related to the Shincheonji religious group in Korea. In the secretive Shincheonji religious group, it is believed that the group's founder and leader can interpret the secret metaphors in the Holy Bible [6]. The members of the group believe that their spirit and bodies are immortal [7]. This belief may have led to their behaviour of not approaching public health authorities when they had COVID-19-related symptoms (e.g., fever or cough) and to their uncooperative attitude towards epidemiological investigations [8]. This is likely to have contributed to the delayed confirmation of cases, despite the massive nationwide public health campaign regarding COVID-19 that was implemented in early February 2020. A previous study demonstrated that early detection of possible cases and prompt interventions are important to help preventing a large number of cases [9]. Therefore, this delay may have resulted in the broad spread of COVID-19 related to the Shincheonji religious group. In this group, worshippers kneel on the floor and hold worship services with a distance of about $10 \mathrm{~cm}$ between worshippers. Furthermore, they usually share lunch boxes after the worship $[10,11]$. Therefore, the method of worship may have also contributed to the transmission of COVID-19.

In our study, fever and cough were the most common symptoms, which is consistent with findings of previous studies conducted in China [12,13]. However, the proportion of cases having fever or cough was lower than that in a previous study of hospitalised patients.

In response to the surge of cases of COVID-19 related to the
Shincheonji religious group, the Korean National Assembly approved the Corona Three Act on February 26, 2020 [14]. This act encoded revisions of the Korean laws on infection prevention, quarantine, and medicine; specifically, the regulations regarding cases of infectious diseases were revised to mandate testing and quarantining of individuals suspected of having COVID-19 by national law. The enactment of the Corona Three Act was a significant event in Korea in that it was the first pangovernmental measure to prevent the spread of infectious diseases.

Our study is the first to analyse the confirmed cases of COVID-19 related to the Shincheonji religious group in Korea, which accounts for the largest proportion of cases of COVID-19 in Korea. However, the cases in our study are a sample collected from the publicly available data related to the Shincheonji religious group, which may have affected the study results. The date of illness onset may differ from the actual date of symptom onset as the data was based on each patient's memory; therefore, it should be noted that this factor may have led to recall bias in our study.

In conclusion, using publicly available data, we estimated a mean delay of 5 days between illness onset to isolation of $\mathrm{CO}$ VID-19 cases among a religious group in Korea. Additional studies to measure the time interval between symptom onset and isolation of cases among different clusters of COVID-19 are needed to help to evaluate the effectiveness of public health interventions against the spread of COVID-19 in the community.

\section{CONFLICT OF INTEREST}

The authors have no conflicts of interest associated with the material presented in this paper.

\section{FUNDING}

None.

\section{ACKNOWLEDGEMENTS}

This research was conducted as a part of the project of Community Medicine and Practice at Department of Preventive Medicine, Konyang University College of Medicine, Daejeon, South Korea. 


\section{AUTHOR CONTRIBUTIONS}

Conceptualization: SR. Methodology: SR. Formal analysis: HJK, HSH, SR. Data curation: HJK, HSH, YHC, HYS, JSP, CYY. Funding acquisition: None. Validation: SR. Writing - original draft preparation: HJK, HSH, YHC, HYS, JSP, CYY, SR. Writing review and editing: SR.

\section{ORCID}

Hyung-Ju Kim https://orcid.org/0000-0001-5626-3721

Hyun-Seong Hwang https://orcid.org/0000-0003-2788-4371

Yong-Hyuk Choi https://orcid.org/0000-0002-0024-7260

Hye-Yeon Song https://orcid.org/0000-0002-4244-7471

Ji-Seong Park https://orcid.org/0000-0002-0722-6788

Chae-Young Yun https://orcid.org/0000-0001-6810-5309

Sukhyun Ryu https://orcid.org/0000-0002-8915-8167

\section{REFERENCES}

1. Ryu S, Chun BC; Korean Society of Epidemiology 2019-nCoV Task Force Team. An interim review of the epidemiological characteristics of 2019 novel coronavirus. Epidemiol Health 2020;42:e2020006.

2. Korea Centers for Disease Control and Prevention. Press release: the updates on COVID-19 in Korea [cited 2020 Mar 16]. Available from: https://www.cdc.go.kr/board/board.es?mid= a30402000000\&bid $=0030$.

3. Nishiura $\mathrm{H}$. Early efforts in modeling the incubation period of infectious diseases with an acute course of illness. Emerg Themes Epidemiol 2007;4:2.

4. Cowling BJ, Park M, Fang VJ, Wu P, Leung GM, Wu JT. Preliminary epidemiological assessment of MERS-CoV outbreak in South Korea, May to June 2015. Euro Surveill 2015;20(25):7-13.

5. Nishiura H. Determination of the appropriate quarantine peri- od following smallpox exposure: an objective approach using the incubation period distribution. Int J Hyg Environ Health 2009;212(1):97-104.

6. Tan L. Shincheonji members helped believer "escape" family. NZME; 2017 Apr 4 [cited 2020 Mar 16]. Available from: https:// www.nzherald.co.nz/nz/news/article.cfm?c_id = 1\&objectid = 11817395.

7. Ahn S. Criticism of Shincheonji doctrine [dissertation]. Seoul: Chongshin University; 2017 (Korean).

8. Borowiec S. How South Korea's coronavirus outbreak got so quickly out of control. TIME; 2020 Feb 24 [cited 2020 Mar 16]. Available from: https://time.com/5789596/south-korea-coronavirus-outbreak/.

9. Ryu S, Kim Bl, Chun BC. An outbreak of respiratory tract infection due to Respiratory Syncytial Virus-B in a postpartum center. J Infect Chemother 2018;24(9):689-694.

10. Hancocks $P$, Seo Y. How novel coronavirus spread through the Shincheonji religious group in South Korea. CNN; 2020 Feb 28 [cited 2020 Mar 16]. Available from: https://edition.cnn.com/ 2020/02/26/asia/shincheonji-south-korea-hnk-intl/index.html.

11. Choi W. "Sit too closely" unique worship contribue the superspread. TV Chosun; 2020 Feb 20 [cited 2020 Mar 16]. Available from: http://news.tvchosun.com/site/data/html_dir/2020/02/ 20/2020022090106.html (Korean).

12. Guan WJ, Ni ZY, Hu Y, Liang WH, Ou CQ, He JX, et al. Clinical characteristics of coronavirus disease 2019 in China. N Engl J Med 2020;382(18):1708-1720.

13. Chen N, Zhou M, Dong X, Qu J, Gong F, Han Y, et al. Epidemiological and clinical characteristics of 99 cases of 2019 novel coronavirus pneumonia in Wuhan, China: a descriptive study. Lancet 2020;395(10223):507-513.

14. Koh J. What is corona three act. Dongascience; 2020 Feb 26 [cited 2020 Mar 16]. Available from: http://dongascience.donga.com/news.php?idx=34612 (Korean). 